\title{
Construção e Dissolução da Conjugalidade: Marcadores e Preditores
}

\author{
Orestes Diniz Neto \\ Universidade Federal de Minas Gerais \\ Terezinha Féres-Carneiro \\ Pontifícia Universidade Católica do Rio de Janeiro
}

\begin{abstract}
RESUMO
O objetivo deste trabalho foi realizar uma revisão dos estudos sobre os marcadores e preditores da construção e dissolução da conjugalidade em uma perspectiva sistêmica, apontando suas implicações para o campo da psicoterapia de casal. As bases de dados consultadas foram PsycINFO e SciELO, utilizando-se as seguintes palavras-chave, em múltiplas combinações, em português e inglês: revisão; terapia de casal; conjugalidade; dissolução da conjugalidade; preditores; marcadores. Foram consultados 132 trabalhos publicados até agosto de 2009. A satisfação conjugal é ressaltada como o principal construto, marcador e preditor envolvido no estudo da formação e manutenção da conjugalidade. São apresentados modelos que descrevem o divórcio como um processo complexo e longo, ressaltando-se que o uso de modelos não lineares pode melhorar a previsibilidade de dissolução da conjugalidade, a partir de fatores psicossociais. Conclui-se que os estudos interacionais trazem importantes contribuições para a clínica de casais, ao sugerir direções e intervenções. Todavia, o simples uso de marcadores e preditores, como variáveis a serem utilizadas em modelos terapêuticos, não tem se revelado útil para melhoria da eficácia terapêutica, pelo fato de serem apenas indicadores de um dado padrão em andamento. Preditores e marcadores seriam, assim, melhor compreendidos como indicadores de processos sistêmicos de estabilidade e mudança.
\end{abstract}

Palavras-chave: formação da conjugalidade; dissolução da conjugalidade, preditores; marcadores; terapia de casal.

\begin{abstract}
Conjugality Construction and Dissolution: Markers and Predictors

The goal of this paper was to review studies about markers and predictors of conjugality construction and dissolution from a systemic perspective, emphasizing the implications for couples' therapy. PsycINFO and SciELO databases were consulted, with the following keywords (in different combinations of Portuguese and English): literature review, couples' therapy, conjugality, conjugality dissolution, predictors, and markers. We consulted 132 studies published until August 2009. Conjugal satisfaction is pointed out as the main concept, marker and predictor involved in both the construction and maintenance of conjugality. Models describing divorce as a complex and long process are presented, emphasizing those non-linear models may improve the possibility of prediction of conjugal dissolution, based on psychosocial factors. We conclude that interactional studies bring important contributions to couples' therapy in suggesting directions and interventions. However, the simple use of markers and predictors as variables in therapeutic models has not been improving therapeutic efficacy, because these are only indicators of certain ongoing patterns. Thus, predictors and markers would be better understood as indicating systemic processes of stability and change.
\end{abstract}

Keywords: construction of conjugality; dissolution of conjugality, predictors; markers; couples' therapy.

A formação e a ruptura do laço conjugal envolvem processos complexos de interações entre os membros do casal, da família e do grupo social (Féres-Carneiro, 2003). Os padrões de relacionamento conjugal têm sido estudados, a partir de indicadores de estabilidade, ruptura, satisfação ou insatisfação do laço conjugal, possibilitando o seu uso como marcadores e preditores da conjugalidade (Goleman, 1998; Gottman, 1998). O estudo e o uso de marcadores, como sinais indicativos da entrada em certa fase de um processo psicoterapêu- 
tico ou de desenvolvimento, e de preditores, como sinalizadores de um aumento de possibilidade de um certo caminho de evolução de um quadro clínico ou de um processo psicológico, são comuns nas teorias psicológicas (Cordioli, 2002; Gottman, 1998; Hoffman, 1995; Rusbult, 1980). Cabe ressaltar que não se trata de variáveis, mas de indicadores de padrões correlacionados a processos. Assim, o objetivo deste trabalho é rever os estudos sobre os marcadores e preditores da construção e dissolução da conjugalidade em uma perspectiva sistêmica, uma vez que tal perspectiva parece fornecer um referencial compreensivo para a complexidade do problema, apontando suas implicações para o campo da psicoterapia de casal.

\section{MÉTODO}

A opção metodológica foi orientada para delinear o desenvolvimento histórico teórico-metodológico dos trabalhos sobre preditores e marcadores da formação e dissolução da conjugalidade. Inicialmente uma pesquisa bibliográfica foi realizada no PsycINFO, em agosto de 2009, com a combinação variada das palavras-chave: review ou revisão; casal ou couple ou marital; preditores ou predictors; marcador ou marker; conjugalidade ou conjugality. Foram localizados 131 trabalhos em inglês, sendo que 59 incluem revisões da literatura, dois descrevem metanálises e um artigo de revisão em português ligado ao tema. Expandindo-se a busca através da SciELO, foram encontrados mais três artigos pertinentes. Estes 134 estudos foram considerados e selecionados pela pertinência ao tema. Em um primeiro momento foram consultadas as revisões que assinalaram os estudos considerados como relevantes teórica e metodologicamente. Estes estudos foram revistos apontando as inovações, introdução de novos métodos, teorias ou técnicas de investigação que influenciaram linhas de inquirição e pesquisa, podendo ser considerados como seminais nas revisões, sendo seguidamente citados. Estes trabalhos são discutidos em termos teórico-metodológicos e epistêmicos em sua implicação para a compreensão da conjugalidade e implicações para psicoterapia de casal. O critério de desenvolvimento temático histórico foi seguido como orientador nesta revisão, pois fornece uma compreensão adequada dos contextos nos quais os desdobramento e propostas de novas perspectivas teórico-metodológicas surgiram. As implicações destes estudos são consideradas a partir de uma abordagem sistêmica que reflete a compreensão epistemológica dos processos psicossociais, como o conjugal, como complexos refletindo uma multiplicidade de aspectos intrincados, inclusive com o olhar do observador e que devem ser abordados por diversos métodos. Assim, adota-se o caminho possível da construção de uma revisão de metapontos de vista - limitados e frágeis - , requisito que diferencia o pensamento simples, que acredita em verdades como realidades independentes do observador. Esta posição permite compreender o entretecer de questões teóricas e metodológicas em diálogo, sem reduzir a uma visão única, mas permitindo a emergência de diferentes perspectivas. Nesta revisão os construtos de satisfação/insatisfação e estabilidade/instabilidade apareceram como eixos temáticos predominantes e orientadores.

\section{Marcadores e preditores da formação da conjugalidade}

A satisfação conjugal é o principal construto, marcador e preditor, envolvido no estudo da construção e manutenção da conjugalidade. De fato, ao seu redor tem sido produzida grande quantidade de pesquisas significativas sobre o laço conjugal. A lógica da justificativa do estudo da satisfação conjugal fundamentase na centralidade da experiência conjugal para o bem-estar do indivíduo e para a família, sendo, ainda, significativo seu impacto na rede social (Bradbury, Finchan \& Beach, 2000). Estes estudos são também importantes por fornecer subsídios para a elaboração de modelos terapêuticos mais adequados ao processo conjugal, respondendo a demandas e questões com maior eficácia e eficiência (Bradbury, Finchan \& Beach, 2000; Gottman \& Notarius, 2002). Além disto, as disfunções conjugais respondem por mais da metade das queixas na clínica psicológica, sendo os modelos propostos para sua compreensão orientados, prioritariamente, pelos estudos das disfunções psicossociais da relação. Assim, são pouco compreendidos os aspectos ligados à satisfação conjugal:

o estudo sistemático da satisfação conjugal permanece vital bem como a importância social de se estudar o como e o porquê casamentos variam na sua qualidade e adequação, apenas por um complexo conjunto de fatores considerados relevantes. (Bradbury, Finchan \& Beach, 2000, p. 965).

Os estudos sobre a satisfação conjugal têm se dispersado em uma vasta e complexa literatura que aborda diversos focos, considerando aspectos demográficos, psicossociais, fatores psicológicos, como traços de personalidade, saúde, psicopatologia, estilos de parentalização, pesquisas desenvolvimentais, e em 
diversas combinações destes enfoques (Gottman, Murray, Swanson, Tyson \& Swanson, 2004). A conceituação de satisfação conjugal tem acompanhado também os vieses teóricos e metodológicos do aconselhamento matrimonial, da psicanálise, da teoria sistêmica e de enfoques psicossociais, herdando suas contribuições e dificuldades. Contudo, alguns aspectos destacam-se em relação à construção de modelos de padrões de desenvolvimento da conjugalidade. É necessário primeiramente rever o desenvolvimento do conceito de conjugalidade e de seu uso nas pesquisas contemporâneas para, em seguida, abordar dois aspectos seminais destes estudos: (1) as pesquisas sobre a interação do casal e das ecologias e contextos socioculturais onde os casais operam, e (2) os processos de avaliação da satisfação conjugal.

O construto da satisfação conjugal foi provavelmente um dos primeiros desenvolvidos, ainda ingenuamente, pelo movimento do aconselhamento matrimonial. Ancorava-se na visão de que um casamento satisfatório seria necessariamente um casamento estável. Durante as primeiras décadas do século XX, o método de estudo sobre a satisfação conjugal foi o autorrelato de cada um dos cônjuges separadamente, procurando traçar uma relação entre personalidade e satisfação conjugal ou de combinações possíveis de estilos que seriam adequados (Broderick \& Schrader, 1991). Um exemplo desta abordagem é o conceito de ajustamento conjugal que foi proposto como "uma adaptação entre marido e mulher ao ponto onde existe companheirismo, concordância sobre valores básicos, intimidade afetiva, acomodação, euforia e certamente outros valores não identificados" (Locke \& Willianson, 1958, p. 569).

Com a ascensão da abordagem psicanalítica, o foco teórico mudou para processos inconscientes de organização psíquica e satisfação recíproca de necessidades e desejos. Durante a emergência do movimento de terapia sistêmica de casal, o foco passou a incluir aspectos da interação conjugal como parte da descrição do que seria funcional em um casal, sempre em relação ao contexto mais amplo da família. O foco deslocou-se de traços ou particularidades do indivíduo para a comunicação enquanto foco de estudo (Watzlawick, Beavin \& Weakland, 1981). Os aspectos interacionais passaram a fazer parte da descrição da estrutura emocional do casal e métodos de estudo específicos foram desenvolvidos, envolvendo a observação do casal:

Estudar o que as pessoas dizem sobre elas próprias não é substituto para estudar como elas se compor- tam. Questionários e escalas de satisfação e insatisfação conjugal têm proporcionado muito pouco. Nós precisamos olhar o que as pessoas fazem umas com as outras. (Raush, Barry, Herti \& Swain, 1974, p. 5)

Assim, na década de 1970, alguns modelos sobre a satisfação conjugal foram propostos. Miller (1976) procurou descrever o conjunto de fatores que apareciam relacionados com a satisfação conjugal. Neste sentido, apontou os antecedentes na socialização como importantes para a realização dos papéis de transição na família, e a duração da convivência como importante para a satisfação conjugal. Esta também seria resultado de uma cadeia de fatores dentre os quais o nível socioeconômico, o número os anos de casado, que marcaria o número de filhos e o espaço para os filhos. A definição do que poderia ser considerado satisfatório em um relacionamento sofreu diversas tentativas de definição, algumas muito gerais, como a proposta por Gray-Little e Burcks (1983), que consideraram que a felicidade conjugal é um sentimento de satisfação subjetivo dos cônjuges em relação ao seu casamento como um todo. Na década de 1980, a diferenciação do relacionamento conjugal como foco de estudo levou ao aparecimento de pesquisas específicas sobre a conjugalidade, nos mais diversos contextos, tais como, estudos epidemiológicos, socioculturais, interacionais com métodos micro e macro-analíticos, qualitativos e quantitativos (Gottman \& Notarius, 2002). As pesquisas dos últimos anos, especialmente na década de 1990, levaram a uma reavaliação do construto de satisfação conjugal (Bradbury e cols., 2000). Estes estudos apontaram que a satisfação conjugal não é a ausência do sofrimento conjugal. Existe um crescente reconhecimento de que satisfação conjugal não é meramente a ausência de insatisfação com o casamento que se tem, como se nota pelo uso, em inglês do termo "nondistress couple" e, em português da expressão "casal funcional". O que leva à satisfação conjugal parece não ser simplesmente o inverso do que leva a sofrimento conjugal.

Discussões na década de 1990 colocaram em relevo os estudos sobre os casamentos que exibem um alto grau de satisfação, em longo prazo, focalizando temas como a ênfase crescente nos padrões interacionais, o reconhecimento das dimensões ecológicas e de aspectos socioculturais. Todos apontando para o desenvolvimento de uma concepção de conjugalidade e de satisfação conjugal que reconheça as diferenças e as especificidades das dimensões únicas do satisfatório e do insatisfatório nos relacionamentos (Cutrona 
1996; Halford, Kelly \& Markman, 1977; Kaslow \& Robison, 1996). Tais estudos revelaram ainda que a satisfação conjugal não é um construto simples, mas que se relaciona com diversos outros. Os primeiros esforços conceituavam a satisfação conjugal como uma avaliação global definindo-a, operacionalmente, como uma única dimensão. Esta definição considera que a insatisfação conjugal reflete uma avaliação do casamento na qual os aspectos negativos estariam salientes e os aspectos positivos ausentes. Por outro lado, satisfação conjugal implicaria em uma avaliação com aspectos negativos ausentes e positivos em destaque. Fincham, Beach e Kemp-Fincham (1997) argumentam que esta é uma avaliação simplista e que avaliações negativas e positivas podem ser conceituadas e consideradas como separadas, ainda que estejam relacionadas. Além disto, a satisfação conjugal não é simplesmente a avaliação da relação conjugal, feita pelos cônjuges, em um dado momento, mas o perceber e o sentir, em relação, um processo ao longo do tempo.

As avaliações realizadas em diversos pontos revelam uma trajetória que reflete as flutuações na qualidade percebida da relação no tempo. Esta trajetória pode ser computada para cada cônjuge, usando múltiplas ondas de dados, e seus paramentos, especialmente sua inclinação ou taxa de mudança no tempo, podem ser examinados em relação a outros fatores. Assim, não é possível compreender totalmente a satisfação conjugal sem a referência a dois ou mais pontos. Essa perspectiva convida a pesquisas com avaliações de múltiplas medidas. Assim, os esquemas de pesquisa tornam-se mais sofisticados, pois se duas medidas supõem, a princípio, modelos lineares, múltiplas medidas necessitam de especificação dos modelos de mudança, muitas vezes não lineares (Wickrama, Lorentz, Conger \& Elder, 1997). E ainda, a satisfação conjugal parece ser mais bem compreendida como uma atitude, do que como uma avaliação, em relação ao cônjuge e à interação conjugal.

Estudos em relação à satisfação conjugal, entendida como atitude, revelam a ideia de que a satisfação pode variar não apenas em grau, mas também em intensidade, na sua associação entre a avaliação (satisfação relatada, por exemplo) e o objeto da avaliação (o casamento ou o cônjuge). Essa associação, ou nível de acessibilidade da atitude, pode ser estudado independentemente da valência da associação. Tais descobertas sugerem que a correlação da satisfação conjugal e o comportamento conjugal podem ser diferentes para aqueles cônjuges com atitudes mais acessíveis, quando comparados com aqueles com atitudes menos acessíveis (Fincham e cols. 1997). Assim, cônjuges com mais acessibilidade de atitude relatariam uma maior satisfação conjugal por estarem engajados em um processo top-down (hierárquico descendente), em relação a cônjuges com menor acessibilidade nos quais predominaria processos bottom-up (hierárquico ascendente) (Bradbury e cols., 2000).

Pesquisadores na tradição cognitivo-social têm examinado a maneira pela qual os cônjuges esforçamse para transformar cognitivamente suas respostas ao comportamento negativo do parceiro, em outras mais benignas. Esta perspectiva sugere que certos contextos estressantes podem exercer um efeito negativo na satisfação com o relacionamento, por interferir com o esforço das transformações cognitivas e, assim, interromper padrões de interações pró-sociais (Yovetich \& Rusbult, 1994). Uma interessante observação encontrada nos modelos cognitivo-sociais de assimilação e efeitos de contraste ressalta que contextos sociais estressantes podem interferir, na satisfação com a relação, apenas até certo ponto, além do qual um aumento no estresse leva a um paradoxal aumento agudo na satisfação com a relação (Teser \& Beach, 1998). Embora estes estudos não tenham sido realizados no contexto de famílias, podem sugerir importantes implicações sobre as relações, o contexto no qual estas ocorrem e a satisfação relatada com as mesmas.

\section{Marcadores e preditores da dissolução da conjugalidade}

A família é o primeiro contexto social de enquadre da conjugalidade e modelos sistêmicos têm usado tanto o ciclo familiar quanto o nexo familiar para descrevê-la e compreendê-la (McGoldrich, 1995). O divórcio é o maior rompimento no processo de vida familiar com importantes impactos para todos os envolvidos, aumentando a complexidade das tarefas desenvolvimentais e existenciais (Stern-Pack \& Manocherian, 1995). O divórcio não se dá, contudo, como um processo simples. Diversos modelos descrevem este processo integrando aspectos significativos. Ahrons (1980), por exemplo, descreve um modelo de cinco fases. Na primeira fase, cognição individual, pelo menos um dos cônjuges está considerando o divórcio e iniciando o processo de separação emocional, mantendo distância através de atividades e envolvimentos separados. Este período é frequentemente caracterizado por estresse, conflitos, amargura, acusações, desvalorização do parceiro, depressão, ansiedade e reiterada ambivalência quanto à decisão. A existên- 
cia de um envolvimento amoroso sexual com um terceiro, nesta fase, pode apressar o processo ou retardálo, uma vez que pode tanto ser fonte de apoio emocional externo, como de culpa acentuada.

No segundo estágio, a metacognição familiar, a decisão tomada ou em elaboração é revelada às famílias extensas e, dependendo do sistema de valores e características da família, esta fase pode acrescentar mais dor e sofrimento aos cônjuges. O cônjuge que toma conhecimento da decisão do outro pode sentir-se aliviado, caso esta decisão também esteja sendo elaborada por ele, ou então pode sofrer profundamente. Quanto mais súbita e inesperada for a decisão, maior o impacto. Na maioria dos divórcios, a decisão de separar é tomada pela esposa, com o marido tipicamente opondo-se à separação. Muitos cônjuges não estão preparados para a situação e experienciam um forte sentimento de baixa autoestima, impotência e desespero.

No terceiro estágio, separação do sistema, ocorre a separação concreta e dependerá muito de como os estágios anteriores foram manejados. Diversos sintomas podem emergir em função do estresse do processo, aumentando o sofrimento e a dificuldade de lidar com a transição. Apesar da raiva e do apego, sempre existe um grau de ambivalência, que pode resultar em múltiplas retomadas da relação e novos rompimentos, tornando mais doloroso ainda o processo de separação. Fatores psicossociais, antecedentes familiares, condições econômicas e culturais podem minimizar ou piorar a situação. Aspectos positivos podem emergir com a sensação de alívio e descoberta de novas possibilidades. As mulheres relatam tipicamente um sentimento maior de melhoria de qualidade de vida pósdivórcio que os homens.

$\mathrm{O}$ quarto estágio, reorganização do sistema, envolve o processo de deixar claras as novas fronteiras. $\mathrm{O}$ processo de separação perturba todos os membros da família. E é necessário reorganizar o funcionamento da família com novas atribuições de responsabilidade e papéis. É importante o papel da rede social neste processo. A continuação dos papéis de pais pode ser um forte elemento de estresse e ser vivido com ambiguidade e tentativas de manipulação do outro cônjuge. Ahrons (1980) encontrou, para casais com filhos, que $25 \%$ tornaram-se adversários furiosos, $25 \%$ sentiam-se sócios zangados, $38 \%$ eram colegas cooperativos e apenas $12 \%$ tornaram-se amigos.

O quinto estágio, redefinição do sistema, começa com uma nova autodefinição da família. Os papéis e fronteiras foram clarificados e todos os membros são incluídos, e os pais vivem bem a paternidade. Quando existe um relacionamento continuado cooperativo entre os ex-cônjuges, a família estabiliza-se rápida e efetivamente.

Este modelo, como outros, descreve a complexa dança que o casal e a família realizam no momento do rompimento conjugal, mas pouco esclarece sobre os processos de ruptura da conjugalidade. Estudos sobre os antecedentes dos rompimentos conjugais têm esclarecido esta questão. Apesar da importância psicossocial da dissolução dos laços conjugais, estudos empíricos sobre seus antecedentes, até a década de 1990, foram bastante raros, sendo feitos, principalmente, sobre fatores epidemiológicos associados à instabilidade conjugal. Foram encontrados fatores como idade: esposas com menos de 18 anos e maridos com menos de 20 (Norton \& Glick, 1976); gravidez préconjugal: tem uma possibilidade duas vezes maior de levar à separação (Furstemberg, 1976); nível de instrução: homens menos instruídos e mulheres mais instruídas têm maior possibilidade de se divorciarem do que homens mais instruídos e mulheres menos (Glick, 1984); salário: mulheres com maior salário têm uma probabilidade maior de se divorciar do que aquelas com salário mais baixo (Ross \& Sanhill, 1975), e quanto maior for o salário da mulher em relação ao do marido, maior será o risco do divórcio (Cherlin, 1979); emprego: quando o emprego do marido é instável ou seu salário oscila em relação ao ano anterior, aumenta o risco do divórcio (Ross \& Sanhill, 1975); nível socioeconômico: as classes menos favorecidas tendem a correr um risco maior (Norton \& Glick, 1976); raça: os negros correm um risco maior que os brancos, e nos casamentos inter-raciais os riscos são ainda maiores (Norton \& Glick, 1976); linha de transmissão transgeracional: o divórcio parece ocorrer mais em determinadas famílias (Mueller \& Pope, 1977).

Estudos que descrevem padrões do relacionamento antecedentes à dissolução do laço conjugal foram raros antes de 1980 (Newcomb \& Bentler, 1981). Morgan, em 1988, analisou dados de um estudo longitudinal de 15 anos, realizado pelo Center of Human Resourse Researsh (1983), com 5.083 mulheres das quais 3.825 estavam casadas. Considerou três tipos de separação conjugal: as que levavam à reconciliação; as de longo termo não resolvidas; e as que levavam ao divórcio. Durante o estudo ocorreram 297 separações. Após três anos, novos dados foram coletados e $29,6 \%$ destas separações haviam levado ao divórcio, $15,8 \%$ à 
reconciliação, e 28,3\% à permanência da separação. As restantes não participaram da pesquisa, por diversas razões, como a viuvez. Apenas os fatores menores ganhos totais familiares e os grupos raciais não brancos foram, significativamente, associados a maiores índices de divórcio e de separação de longo prazo. $\mathrm{O}$ menor nível educacional foi associado ao maior número de reconciliações. Morgan (1988) sugere que o nível educacional mais baixo poderia aparecer associado ao divórcio por estar envolvido no processo de separação, que normalmente precede o divórcio, mas não parece estar associado à tomada de decisão do rompimento definitivo do vínculo conjugal. De fato, poderia levar à reconciliação uma vez que a mulher poderia sentir-se com poucos recursos pessoais para lidar com o divórcio ou separação prolongada. Estudos epidemiológicos forneceram as primeiras abordagens do complexo fenômeno do rompimento conjugal, mas modelos mais adequados deveriam abordar, de modo prospectivo, os preditores da evolução da relação conjugal.

Estudos sobre a conjugalidade e a experiência subjetiva dos casais foram realizados desde a década de 1980, principalmente por meio de entrevistas e questionários. Poucos, no entanto, realizaram testes de consistência e validade dos construtos internos ou apresentaram modelos preditivos e testes de predição. Belsky, Sapnier e Rovine (1983) e Cowan e Cowan (1989) foram os dois únicos estudos na década de 1980 que, ao analisar a transição do casal para a parentalidade, tentaram predizer os resultados da satisfação conjugal. Em relação ao rompimento conjugal, quatro estudos foram publicados na década de 1980: Bentler e Newcomb (1978), Block, Block e Morisson (1981), Constantine e Bahr (1980) e Kely e Conley (1987) - todos se baseando apenas em fatores avaliados de cada cônjuge. Estes estudos obtiveram baixa, ou nenhuma, correlação com predições de divórcio, resultando em quadros teóricos empobrecidos. O modelo de análise foi linear, resultando em pouco valor preditivo.

Bentler e Newcomb (1978), já no final dos anos setenta, notaram que casais, que permaneciam casados eram mais similares em relação à idade, interesse em artes e atratividade do que casais separados ou divorciados. Homens separados, neste estudo, descreviam a si mesmos como mais invulneráveis, extrovertidos e envelhecidos do que aqueles que permaneciam casados. As mulheres separadas ou divorciadas descreviam a si mesmas como menos conscientes de suas roupas e menos agradáveis que as casadas. Constantine e Bahr
(1980), em um estudo longitudinal de seis anos, descobriram que os homens que haviam se separado ou se divorciado tinham maior "orientação interna", em uma escala de "lócus de controle", que os indivíduos que permaneceram casados. Block, Block e Morrison (1981) estudaram 57 famílias, com crianças de três anos e meio, discriminando-as em famílias intactas e divorciadas, e, após dez anos de acompanhamento, encontraram diferenças nos estilos de escuta às crianças, no sentido de que as primeiras apresentavam uma melhor qualidade. Kelly e Conley (1987), utilizando escalas de familiaridade de personalidade, em um estudo longitudinal de 35 anos de estabilidade conjugal, relataram que os homens que permaneceram casados eram mais convencionais, menos neuróticos, tinham maior controle de impulsos que aqueles divorciados. Dados similares foram encontrados para as mulheres, com a descoberta adicional de que as que permaneceram casadas possuíam um maior fechamento emocional e menor tensão em relação à sua família de origem.

Em seu conjunto, esses estudos sugerem que os casais que permaneceriam casados seriam os mais conservadores, com os cônjuges mais similares em interesses e menos neuróticos. Tais resultados são limitados em vários aspectos e questionáveis metodologicamente. Poderiam estar descrevendo apenas um tipo de casal específico que tende a permanecer casado, enquanto que a emergência de traços neuróticos poderia ser sinal de padrões disruptivos na relação, já presentes há mais tempo. Nesses estudos, o uso de técnicas lineares de análise, como análise da variância, por exemplo, tem também sido questionado, pois padrões mais complexos poderiam estar envolvidos.

Pesquisas têm sido realizadas com o objetivo de expandir a previsibilidade de modelos do relacionamento conjugal. Rogge (2003), por exemplo, desenvolveu algoritmos não lineares para explorar e prever os fatores preditores de discórdia conjugal e de divórcio, nos primeiros estágios do casamento. Usando um modelo de dois fatores, neuroticismo e hostilidade, este autor foi capaz de prever, em uma amostra de 85 casais de Munique, Alemanha, com $65 \%$ de precisão, quais casais permaneceriam casados e quais se separariam nos primeiros cinco anos de casados. A presença de comunicação negativa, nos casais restantes, previa insatisfação com o casamento. Rogge, em 2003, estendeu sua análise a 480 casais recém-casados de Bufallo (Nova Iorque, EUA) e 172 casais de Los Angeles (Califórnia, EUA). Medidas de agressão física e verbal foram usadas para predizer o divórcio e 
medidas de estresse, traços de raiva e comunicação empobrecida para predizer insatisfação conjugal, em três anos do estudo. Os casais da amostra de Búfalo foram classificados com $52 \%$ de precisão no caso de divórcio. Quando, no algoritmo, incluiu-se o grupo racial este nível subiu para 57\%. Quando foram incluídas as presenças ou ausências de crianças, de modo diferenciado, no algoritmo este nível subiu para $64 \%$. $\mathrm{O}$ algoritmo resultante foi aplicado prospectivamente à amostra de casais de Los Angeles, tendo sido capaz de predizer com $60 \%-66 \%$ de precisão o desenvolvimento da relação conjugal em três anos. Estes resultados trouxeram importantes contribuições, pois, refinaram a compreensão de como fatores envolvidos podem ser utilizados para prever o desenvolvimento da relação conjugal, nos primeiros anos de casamento. Além disto, melhoraram os modelos de predição incluindo variáveis como grupo racial e parentalidade anterior ao casamento - apresentaram um algoritmo não linear com múltiplas variáveis para predição, e forneceram a primeira evidência de validação prospectiva de algoritmos preditivos com fatores psicossociais.

É importante considerar que o uso de modelos não lineares pode melhorar a previsibilidade de divórcio, a partir de fatores psicossociais. Mas cabe ressaltar que modelos preditivos em relação ao divórcio padecem de dificuldades metodológicas inerentes ao seu objeto. Considerando o aumento da taxa de divórcio no ocidente, que chega a mais de $50 \%$ dos casais, e que mais da metade dos divórcios ocorrem até os quatro primeiros anos de casamento, é de se esperar que modelos atinjam níveis melhores de previsibilidade.

Gottman e Levenson (1992) notam o pouco conhecimento obtido por estudos epidemiológicos, que não fornecem nenhuma teoria coerente sobre como casais interagiam e os seus riscos de rompimento de relações conjugais. Lembram ainda que a insatisfação conjugal pode ser um processo independente do rompimento do vínculo conjugal, como já fora apontado por Laederer e Jackson (1968). Outros estudos (Blomm, White \& Archer, 1978; Emerly, 1988; Levinger \& Moles, 1979) forneceram poucos dados sobre os padrões de interação conjugal que levavam a separação. Tal falha decorre, em parte, por tratar a separação e o divórcio como variáveis independentes, mais do que como variáveis dependentes, preocupando-se mais com os efeitos do divórcio do que com seu processo antecedente. Estes estudos podem ser criticados, pois tratam de eventos psicossociais como variáveis independentes que podem estar sistemicamente relacionados. Abordagens interacionais fornecem propostas voltadas para a análise do sistema conjugal em andamento. Elas foram sugeridas como uma abordagem útil para compreensão dos padrões de funcionamento de um casal, desde a emergência da terapia sistêmica de família (Watzlawick e cols. 1981). No entanto, apenas tardiamente, nas décadas de 1980 e 1990, é que se realizaram estudos sobre os padrões de interação em andamento. As razões para tanto são compreensíveis.

Primeiro, a psicologia, durante muitos anos identificou o seu objeto com o estudo do indivíduo e seus processos específicos como memória, inteligência, psicopatologia dentre outros, e não com interações em andamento. Em segundo lugar, as primeiras pesquisas sobre as raízes psicopatológicas, ou disfuncionalidades psicológicas, como parte do sistema em andamento, resultaram pouco convincentes. Em artigo de revisão sobre o tema, Frank (1965) concluiu que não havia relações entre a psicopatologia e os processos familiares, sendo as disfunções melhor compreendidas como perturbações causadas por problemas individuais. O problema inicial parece estar relacionado ao fato de as teorias e pesquisas, propostas pelos teóricos sistêmicos, terem falhado nos desenhos experimentais pela inadequação dos métodos de avaliação e da análise estatística usada. No entanto, novos métodos de pesquisa têm sido desenvolvidos. As características destes estudos têm sido marcadas por uma cuidadosa abordagem psicrométrica, particularmente quanto à validade externa dos construtos, com um enquadre multimetodológico, e uma ênfase em técnicas de observação quantitativas e qualitativas. Assim, estudos longitudinais prospectivos e preditivos, em uma abordagem desenvolvimental, têm sido realizados, tendo a vida familiar como enquadre.

Esta abordagem tem gerado, nas últimas décadas, importantes resultados, embora as implicações para a terapia de casal e de família tenham sido pouco exploradas (Diniz-Neto, 2005). Alguns dos estudos mais significativos apresentam tanto tendências metodológicas como resultados relevantes. Gottman e Levenson (1992) identificaram processos de interação conjugal que puderam ser descritos em um modelo de "tipo cascata" da dissolução matrimonial, descrevendo o processo de separação em uma hipótese simples que envolve baixa satisfação conjugal, levando à consideração, por um ou ambos os cônjuges, da possibilidade de se separar, levando à separação real, seguida de reconciliação e, levando ao divórcio. Uma importante mudança metodológica, introduzida neste estudo, foi a observação em laboratório da interação conjugal real, em uma entrevista com tarefas estruturadas, após o 
casal ter passado pelo menos 8 horas sem interagir. Nesta entrevista, o casal deveria, em três momentos sucessivos, durante 15 minutos cada, discutir sobre: os fatos do dia; uma situação problemática do casal; e uma situação agradável de concordância mútua. Após cada discussão, havia um intervalo de 5 minutos, no qual ocorria uma gravação da interação do casal e, além disso, eram realizadas medidas fisiológicas. Cada cônjuge foi convidado, dias depois, a assistir separadamente à gravação dos períodos de intervalo da entrevista, e a indicar em um dial com nove posições, que iam de muito desagradável a muito agradável, com neutro no meio da escala, como se sentia no episódio da interação.

Os valores desta medida subjetiva de satisfação/insatisfação eram acumulados sucessivamente em relação à interação em andamento, gerando padrões predominantemente positivos ou negativos. Isto permitiu separar os casais em dois grupos: "regulados", na qual a medida era predominantemente positiva, e "não regulados", com o predomínio de medidas negativas. Este procedimento foi repetido, mais uma vez, quatro anos mais tarde, e também foram coletados dados da vida do casal, através de entrevistas suplementares e questionários. Os casais "não regulados" diferiam dos "regulados" por apresentarem: problemas conjugais mais severos; menor satisfação conjugal; saúde mais frágil; menor amplitude de pulsação cardíaca nos dedos durante as interações; maior número de interações negativas; expressões emocionais mais negativas em situações de conflito; menos expressões emocionais positivas nas interações; mais inflexibilidade e fuga nas discussões; maior atitude defensiva; maior risco de dissolução conjugal. A análise dos dados forneceu apoio à hipótese de um modelo em cascata no qual: casais que se divorciaram tendiam a ter se separado e reconciliado antes; casais que se separaram e reconciliaram relatavam ter considerado a dissolução antes; e casais que consideraram a dissolução, apresentavam menor satisfação conjugal na segunda e na primeira medida, que os casais que permaneceram casados. Foi testado outro modelo linear alternativo para avaliar a adequação do modelo em cascata, sendo realizado um teste de análise da variância entre os dados. Este modelo alternativo não se ajustou aos dados, sugerindo que o modelo em cascata aproximava-se melhor da descrição da dinâmica conjugal disfuncional (Gottman \& Levenson, 1992).

O método introduzido por Gottman e Levenson (1992) gerou diversos trabalhos voltados para o desenvolvimento de modelos não lineares para o estudo do casamento (Gottman, 1998; Gottman \& Levenson, 1999a, 1999b; Gottman, Swanson \& Murray, 1999); de padrões de relacionamento conjugal (Gottman \& Levenson, 1999a, 1999b; Parsupathi, Carstensen, Levenson \& Gottman, 1998); e de preditores de dissolução conjugal e de insatisfação conjugal (Carrere \& Gottman, 1999; Gottman \& Levenson, 1999a; Gottman, Murray, Swanson, Tyson \& Swanson, 2004), entre outras contribuições. Contudo, apesar de estes estudos lançarem novas luzes sobre preditores e padrões de relacionamento conjugal, devemos lembrar que

Conhecer alguns dos fatores causais da dissolução conjugal não é suficiente para construirmos um modelo da conjugalidade funcional. Descobertas recentes indicam que casamentos estáveis e satisfatórios são baseados em uma série de processos conjugais e comportamentos que são mais que simplesmente a ausência de processos disfuncionais. (Carrere \& Gottman, 1999, p. 3)

\section{CONSIDERAÇÕES FINAIS}

Os estudos interacionais trazem importantes contribuições para a clínica de casais ao sugerir direções e intervenções. É necessário, pois, descrever estes fatores em uma teoria coerente que permita não só a previsão de possíveis riscos para o laço conjugal, mas que esclareça sua relação com os processos psicossociais, possibilitando, ainda, seu uso na avaliação das mudanças realizadas em intervenções psicoterapêuticas. O simples uso de marcadores e preditores, como variáveis a serem manipuladas em modelos terapêuticos, não tem se revelado útil para melhora da eficácia terapêutica (Gottman, 1998). Intervenções diretas sobre os preditores têm-se mostrado insuficientes, e clientes revelam que muitas vezes são sentidas como artificiais e estranhas à sua subjetividade (Mahoney, 1991, 1997). Talvez este resultado possa ser explicado pelo fato de marcadores e preditores simplesmente não serem variáveis, mas apenas indicadores de um dado padrão em andamento, que não pode ser alcançado pela simples ativação destes. Preditores e marcadores seriam, assim, melhor compreendidos como indicadores de processos sistêmicos de estabilidade e mudança. Outra questão importante é que, como indica Hoffman (1995), entre outros autores, sistemas disfuncionais apresentam padrões de maior rigidez e consequentemente padrões mais previsíveis. Assim, paradoxalmente, quanto mais disfuncional for um casal mais poderíamos descrever modelos previsíveis de suas interações. 
Sugerir direções de mudanças não é o mesmo que realizá-las. No entanto, estes estudos não são diretamente deriváveis para psicoterapia, embora possibilitem a construção de modelos de relacionamento conjugal que poderiam fundamentar intervenções terapêuticas e modelos de terapia de casal. Tais trabalhos constituem uma importante contribuição, pois a previsão de linhas de desenvolvimento de casais, utilizando modelos e conceitos derivados de construções eminentemente clínicas não tem possibilitado, até agora, prever as linhas de evolução do ciclo conjugal. Estes estudos têm implicações éticas, pois ser capaz de inferir o fluxo provável não significa que tal informação deva ser passada aos clientes ou usada com força de verdade. Mas pode ser utilizada pelo terapeuta como linha de avaliação sobre o sucesso de suas intervenções, possibilitando o reconhecimento de mudança na dinâmica do casal, ajustando as intervenções, facilitando o relacionamento e o processo terapêutico.

Resumidamente, a construção de um modelo de terapia de casal, considerando estes estudos, deverá incluir uma compreensão sistêmica do processo de mudança, explicitando os procedimentos de intervenção e integrando as diferentes questões da subjetividade dos clientes, respondendo às demandas em diversos pontos do ciclo conjugal e familiar. Este parece ser o maior desafio.

\section{REFERÊNCIAS ${ }^{1}$}

*Ahrons, C. R. (1980). Redefing the divorce family: A conceptual framework. Social Work, 25, 437-441.

*Belsky, J. Spanier, G. B., \& Rovine, M. (1983). Stability and change in marriage across the transition to parenthood. Journal of Marriage and the Family, 45, 567-577.

*Bentler, P. M., \& Newcomb, M. D. (1978). Longitudinal study of marital success and failure. Journal of Consulting and Clinical Psychology, 46, 1053-1070.

*Block, J. H., Block, B., \& Morrison, A. (1981). Parental agreement-disagreement on child-rearing and gender related personality correlates in children. Child Development, 52, 965-974.

*Blomm, B. L., White, S. W., \& Archer, S. J. (1978). Marital disruption as a stressor: A review and analysis. Psychological Bulletin, 6, 564-573.

*Bradbury, T. N., Finchan, F. D., \& Beach, S. R. (2000). Research on the nature and determinats of marital satisfaction: A decade in review. Journal of Marriage and Family, 62, 964-980.

*Broderick, C. B., \& Schrader, S. S. (1991). The history of professional marriage and family therapy. Em A. S. Gurman \& D. P. Kniskern (Orgs.), Handbook of family therapy (pp. 3-40). New York: Brunner/Mazel.

*Carrere, S., \& Gottman, J. M. (1999). Predicting the future of marriages. Em E. M. Hetherington (Org.), Coping with divorce, single parenting, and remarriage: A risk and resiliency perspective (pp. 3-22). Mahwah, NJ: Lawrence Erlbaum.
*Cherlin, A. J. (1979). Marriage, divorce, remarriage. Cambridge, MA: Harvard University Press.

*Constantine, J. A., \& Bahr, S. J. (1980). Locus of control and marital stability: A longitudinal study. Journal of Divorce, 4, 11-22.

Cordioli, A. V. (2002). Como atuam as psicoterapias. Em A. V. Cordioli (Org.), Psicoterapias atuais (pp. 35-46). Porto Alegre; Artes Médicas.

*Cowan, P. A., \& Cowan, C. (1989). Marital relationship, parenting style, and the child's development at the age of three. Voprosy-Psikhologiii, 4, 110-118.

*Cutrona, C. (1996). Social support in couples. Thousand Oaks, CA: Sage.

*Diniz-Neto, O. (2005). Conjugalidade: Proposta de um modelo construcionista social de terapia de casal. Tese de doutorado não-publicada. Pontifícia Universidade Católica do Rio de Janeiro.

*Emerly, R. E. (1988). Marriage, divorce and children's adjustment. Newbury Park, CA: Sage.

*Féres-Carneiro, T. (2003). Construção e dissolução do laço conjugal em terapia de casal. Em T. Féres-Carneiro (Org.), Família e casal: Arranjos e demandas contemporâneas (pp. 109-127). Rio de Janeiro: Editora PUC-Rio.

*Finchan, E. D., Beach, S. R., \& Kemp-Finchan, S. (1997). Marital quality: A new theoretical perpective. Em R. J. Sternberg \& M. Hojat (Orgs.). Satisfaction in close relationships (pp. 275-304). New York: Guilford Press.

*Frank, G. H. (1965). The role of the family in the development of psychopathology. Psychological Bulletin, 64, 191-205.

*Furstemberg, F. F. J. (1976). Premarital pregnancy and marital stability. Journal of Social Issues, 32, 36-43.

*Glick, P. C. (1984). Marriage, divorce and living arrangements: Prospective changes. Journal of Family Issues, 3, 32-41.

Goleman, D. (1998). Inteligência emocional (M. Santarrita, Trad.). Rio de Janeiro: Objetiva.

*Gottman, J. M. (1998). Psychology and the study of marital process. Annual Review of Psychology, 49, 169-197.

*Gottman, J. M., \& Levenson, R. W. (1992). Marital process predictive of latér dissolution: Behavior, physiology and health. Journal of Personality and Social Psychology, 63(2), 221-233.

*Gottman, J. M., \& Levenson, R. W. (1999a) What predicts change in marital interction over time? A study of alternative medicine. Family Process, 38(2), 143-158.

*Gottman, J. M., \& Levenson, R. W. (1999b) Rebound from marital conflict and divorce prediction. Family Process, 38(3), 287-292.

*Gottman, J. M., Murray, J. D., Swanson, C. C., Tyson, R., \& Swanson, K. R. (2004). The mathematics of marriage: Dynamic nonlinear models. London: MIT Press.

*Gottman, J.M. \& Notarius, C.I. (2002). Marital research in the 20 th century and a agenda for the 21 th century. Family Process, 41 (2), 159-198.

*Gottman, J. M, Swanson C. \& Murray, J. (1999c). The mathematics of marital conflict: Dynamic mathematical nonlinear modeling of newlywed marital interaction. Journal of Family Psychology , 13(1), 3-19.

*Gray-Little B., \& Burks, N. (1983). Power and satisfaction in marriage: A review and a critique. Psychological Bulletin, 93, 513-538 
*Halford, W. K., Kelly, A., \& Markman, H. J. (1997). The concept of a heathy marriage. Em W. K. Halford \& H. J. Markman (Orgs.), Clinical handbook of marriage and couple interventions (pp. 3-12). New York: Wiley.

Hoffman, L. (1995). O ciclo de vida familiar e a mudança descontínua. Em B. Carter \& M. McGoldrick (Orgs.), As mudanças no ciclo de vida familiar (M. A. V. Veronese, Trad.) (pp. 8496). Porto Alegre: Artes Médicas.

*Kaslow, F., \& Robinson, J. A. (1996). Long term satisfying marriages: Perceptions of contributing factors. American Journal of Family Therapy, 24, 153-170.

*Kelly, L. E., \& Conley, J. J. (1987). Personality and compatibility: A prospective analysis of marital stability and marital satisfaction. Journal of Personality \& Social Psychology, 52, 27-40.

*Laderer, W. J., \& Jackson, D. D. (1968). The mirages of marriage. New York: Norton.

*Levinger, G., \& Moles, O. C. (1979). Divorce and separation: Context, causes and consequences. New York: Basic Books.

*Locke H. J., \& Willianson, R. C. (1958) Marital adjustment: A factor analysis study. American Sociological Review, 23, 562569.

Mahoney, M. J. (1991). Human change process: The scientific foundations of psychotherapy. New York: Basic Books.

Mahoney, M. J. (1997). Evolução contínua das ciências cognitivas e psicoterapias. Em R. A. Neimeyer \& M. J. Mahoney (Orgs.), Construtivismo em psicoterapia (pp. 38-59). Porto Alegre: Artes Médicas.

McGoldrick, M. (1995). A união das famílias através do casamento: O novo casal. Em B. Carter \& M. McGoldrick (Orgs.), As mudanças no ciclo de vida familiar (pp. 184-205). Porto Alegre: Artes Médicas.

*Miller, B. C. (1976). A multivariate developmental model of marital satisfaction. Journal of Marriage and Family, 38, 643657.

*Morgan, L. A. (1988). Outcomes of marital separation: A longitudinal test of predictors. Journal of Marriage and Family, 50, 493-498.

*Mueller, C. W., \& Pope, H. (1977). Transmission between generations. Journal of Marriage and Family, 39, 114-123.

*Newcomb, M. D., \& Bentler, P. M. (1981). Marital breakdown. Em S. Duck \& R. Gilmour (Orgs.), Personal relationships (pp. 57-94). San Diego: Academic Press.

Nota:

${ }^{1}$ Referências precedidas de um asterisco indicam estudos incluídos na revisão.

\section{Sobre os autores:}

Orestes Diniz Neto: Psicólogo, Doutor em Psicologia (Psicologia Clínica) pela Pontifícia Universidade Católica do Rio de Janeiro (2005), professor adjunto da Universidade Federal de Minas Gerais.

Terezinha Féres-Carneiro: Psicóloga, Doutora em Psicologia (Psicologia Clínica) pela Pontifícia Universidade Católica de São Paulo (1981), Pós-doutorada pela Universidade de Paris V (1988), professora titular da Pontifícia Universidade Católica do Rio de Janeiro.

Endereço para correspondência: Universidade Federal de Minas Gerais - Campus Pampulha - FAFICH - Departamento de Psicologia - Av. Antônio Carlos, 6.627 - 31270-901 Belo Horizonte/MG. Endereço eletrônico: orestesdneto@ufmg.br.
*Norton, S., \& Glick, P. C. (1976). Marital instability: Past, present, and future. Journal of Social Issues, 32, 5-20.

*Parsupathi, M., Carstensen, R., Levenson, W. R., \& Gottman, J. M. (1998). Responsive listening in long married couples: A psycholinguistic perspective. Journal of Nonverbal Behavior, 23(2), 173-193.

*Raush, H. L., Barry, W. A., Hertl, R. K., \& Swain, M. A. (1974). Communication, conflict, and marriage. San Francisco: JosseyBass.

*Rogge, R. D., Jr. (2003). An examination of the predictors of discord and divorce in the early stages of marriage. Dissertation Abstracts International - Section B: Sciences and Engineering, 64 (1-b), 429.

*Ross, H. L., \& Sanhill, I. V. (1975). Time of transition: The growth of family headed by women. Washington: Urban Press.

*Rusbult, C. E. (1980). Commitment and satisfaction in close relationship: An interdependence analysis. Journal of Social and Personal Relationship, 10, 175-203.

Stern-Peck, J., \& Manocherian, J. (1995). O divórcio no ciclo de vida familiar. Em B. Carter \& M. McGoldrick (Orgs.), As mudanças no ciclo de vida familiar: Uma estrutura para a terapia familiar (M. A. V. Veronese, Trad.) (pp. 291-320). Porto Alegre: Artes Médicas. (Original publicado em 1989)

*Tsser, A., \& Beach, S. R. H. (1998). Life events, relantionships quality and depression: An investigation of judgment discontinuity in vivo. Journal of Personality and Social Psychology, $74,36-52$.

*Watzlawick, P. Beavin, J., \& Jackson, D. (1981). Pragmática da

*Wickrama, K. A., Lorenz, F. O., Conger, R. D., \& Elder, G. H., Jr. (1997). Marital quality and physical ilness: A latent growth curve analysis. Journal of Marriage and the Family, 59, 143155.

Yovetich, N. A., \& Ruabult, C. E. (1994). Accommodative behavior in close relantionships: Exploring transformation of motivation. Journal of Experimental Social Psychology, 30, 138-164.

Recebido: 14/09/2009

Última revisão: 05/05/2010 Aceite final: 20/06/2010 comunicação humana (A. Cabral, Trad.). São Paulo: Cultrix. 